\title{
Avaliação do perfil morfofuncional de recrutas ingressantes em uma unidade militar da força aérea brasileira
}

\author{
Evaluation of recruits morphofunctional profile from \\ a military unit of brazilian air force
}

\author{
Leandra Cristina Benetti Campos, 1,2 Fábio Angioluci Diniz Campos, ${ }^{1,2}$ Thiago Augusto Rochetti Bezerra, ${ }^{1}$ \\ Ídico Luiz Pellegrinotti \\ 'Academia da Força Aérea, Pirassununga, SP, Brasil. \\ 2Faculdade de Ciências da Saúde, Universidade Metodista de Piracicaba. Piracicaba, SP, Brasil.
}

Recebido em: 26/05/2016 / Aceito em: 30/06/ 2016

leandrabenetti@gmail.com

\section{RESUMO}

Objetivo: analisar o perfil morfofuncional de recrutas de uma unidade militar da Força Aérea Brasileira. Método: a amostra foi composta por 139 recrutas voluntários com idade entre 18-19 anos. Foram avaliadas variáveis antropométricas (peso corporal, estatura, índice de massa corporal, circunferências de braço, cintura e quadril, relação cintura/quadril, dobras cutâneas (tríceps, suprailíaca e abdominal) e percentual de gordura) além de testes de resistência abdominal, resistência de membros superiores e teste de corrida de 12 minutos. Os dados foram submetidos a procedimentos estatísticos descritivos, contendo média, desvio padrão, variação e percentual. Resultados: os resultados indicam $14,1 \pm 7,2 \%$ de gordura corporal, destacando que, de acordo com a classificação do estado nutricional do índice de massa corporal, 75,54\% dos avaliados são eutróficos, 9,35\% baixo peso corporal e $15,11 \%$ sobrepeso ou obesidade. O VO $\mathrm{VO}_{2 \max }$ observado foi de $38,16 \pm 7,06 \mathrm{ml} \cdot \mathrm{kg}^{-1} \cdot \mathrm{min}^{-1}$. Considerações finais: considera-se crítico a parcela da amostra ( 25\%) esta com peso inadequado (baixo peso ou sobrepeso) e a baixa capacidade aeróbia dos sujeitos.

Palavras-chave: Militares; Composição Corporal; Aptidão Física.

\section{ABSTRACT}

Objective: study was to analyse the functional morphology of recruits of a military unit of the Brazilian Air Force. Method: the sample consisted of 139 recruits volunteers aged 18-19 years. This study met the standards for conducting research on human subjects, the National Health Council in 1996 and was approved by the local ethics committee. Anthropometric variables were assessed (body mass, height, body mass index, arm circumference, waist and hip, waist / hip ratio, skinfold, (triceps, suprailiac and abdominal) and body fat percentage) and abdominal strength tests, strength of the upper limbs and 12 minute running test. Descriptive statistical procedures were performed with mean, standard deviation, variance and percentage. Results: indicate $14.1 \pm 7.2 \%$ body fat, noting that according to the nutritional status, body mass index $75.54 \%$ of the individuals are eutrophic, 9.35\% lower body weight and $15.11 \%$ overweight or obese. $\mathrm{VO}_{2 \max }$ observed was $38.16 \pm 7,06 \mathrm{ml} . \mathrm{kg}^{-1} \cdot \mathrm{min}^{-1}$. Closing remarks: it is considered disturbing the portion of the sample ( 25\%) with this inadequate weight (underweight or overweight) and the low aerobic capacity of the subjects of this study.

Keywords: Military; Body Composition; Physical Fitness. 


\section{INTRODUÇÃO}

A carreira dos soldados, nas organizações militares, caracteriza-se por um curso inicial de recrutas, passando por um período de adaptação ao meio militar, para posteriormente serem intitulados soldados. Os recrutas são preparados para a carreira militar, garantindo a segurança das organizações militares e da sociedade. Dentro deste contexto, devem ser preparados fisicamente para estarem aptos às missões a ele impostas.

Importante destacar que níveis adequados de aptidão física e de atividade física têm sido indicados como fatores de proteção para inúmeras doenças e como promotores de qualidade de vida. ${ }^{1,2}$ De acordo com Neves, ${ }^{3}$ o perfil nutricional dos brasileiros tem revelado que as prevalências de sobrepeso e obesidade cresceram de maneira importante nos últimos trinta anos. No meio militar, essas condições não são somente fatores de saúde física, mas também de sobrevivência caso seja necessário seu emprego operacional. A carreira militar exige de seus profissionais uma condição física mínima, suficiente para o desempenho de funções militares específicas em tempo de paz e de guerra. ${ }^{4}$ Estima-se que $8,8 \%$ dos homens adultos brasileiros são obesos, com uma maior prevalência nas regiões Sul e Sudeste do país. ${ }^{5}$ Ressalta assim que a falta de atividade física e a obesidade constituem um grande fator de risco para doenças como hipertensão, diabetes entre outras.

A capacidade aeróbia mensurada através do $\mathrm{VO}_{2 \max }$ depende dos componentes cardiovasculares, respiratórios, hematológicos e de mecanismos oxidativos do musculo em exercício. ${ }^{6}$ Assim, é considerado o melhor indicador da condição cardiovascular tornando-o importante parâmetro preditivo de morbidades associadas. ${ }^{7}$ Estudos apontam que os níveis de aptidão cardiorrespiratória estão associados com adiposidade total e abdominal e os sistemas muscular e cardiorrespiratório são associados com principais de fatores de risco para doenças cardiovasculares. ${ }^{8}$ É extremamente importante que os militares da ativa sejam saudáveis, estejam prontos para as atividades operacionais e ações de combate. ${ }^{9}$ Assim, torna-se necessário o entendimento de qual a característica morfofuncional no momento de sua chegada a Organização Militar.

Em especial, podem-se encontrar estudos nacionais que analisaram o perfil nutricional de militares. No entanto, são escassos os estudos que envolvem jovens recrutas nas Forças Armadas, particularmente na Força Aérea Brasileira. Neste sentido, o objetivo deste estudo foi determinar o perfil morfofuncional em recrutas de uma unidade militar da Força Aérea Brasileira. A hipótese de nosso estudo é que o perfil dos recrutas segue uma tendência brasileira em relação às variáveis analisadas, de acordo com a faixa etária.

\section{MÉTODO}

A pesquisa teve caráter descritivo transversal. A amostra foi composta por 139 recrutas do sexo masculino pertencentes a uma unidade da Força Aérea Brasileira, com idade entre 18 e 19 anos. Os dados foram coletados com os sujeitos da primeira turma do curso de formação de soldados no ano de 2016. Os partici- pantes foram informados dos objetivos e procedimentos do estudo, assinando o Termo de Consentimento Livre e Esclarecido, estando livres para interromperem a pesquisa no momento em que desejassem. O presente estudo atendeu as normas para a realização de pesquisa em seres humanos, do Conselho Nacional de Saúde e foi aprovado pelo comitê de ética local.

As variáveis antropométricas investigadas foram estatura (mts), massa corporal $(\mathrm{kg})$, dobras cutâneas (tríceps, suprailíaca e abdômen - $\mathrm{mm}$ ), circunferências (braço, cintura e quadril - cm), percentual de gordura, massa corporal magra e massa corporal gorda. Todas as medidas foram coletadas por dois avaliadores, com erro inter-avaliador de 2,2\%, considerado aceitável. ${ }^{10}$ Para a coleta da estatura, foi utilizado um estadiômetro marca Wiso, em que os sujeitos permaneceram em posição ereta, pés descalços, encostados em uma superfície plana e vertical, calcanhares unidos, cabeça ajustada ao plano de Frankfurt e inspiração profunda. A pesagem foi realizada com os recrutas descalços, vestidos com short em uma balança digital marca Welmy com precisão de $50 \mathrm{~g}$.

Para a coleta das dobras cutâneas, foi utilizado um adipômetro marca Cescorf. Para a verificação do percentual de gordura corporal, foi aplicado o protocolo proposto por Guedes, ${ }^{11}$ para a determinação da densidade corporal e a equação de Siri (1961), para a determinação do percentual de gordura corporal. A massa corporal magra (MCM) e a massa corporal gorda (MCG) foram calculadas de acordo com as seguintes equações: $\mathrm{MCM}=$ massa corporal $-\mathrm{MCG}$ e $\mathrm{MG}=$ massa corporal x (\% gordura/100).

De acordo com os resultados antropométricos, foram realizados cálculos de índice de massa corporal $\left.(\text { IMC = massa corporal/(estatura })^{2}\right)$ e relação de circunferência de cintura e de quadril $(\mathrm{RCO}=$ Circunferência de cintura / circunferência de quadril). Os índices como o IMC e a RCQ são importantes indícios de sobrepeso e obesidade por serem indicadores práticos, de baixo custo e reproduzíveis. ${ }^{3} \mathrm{O}$ IMC tem sido utilizado como indicador válido do estudo nutricional em diversos grupos populacionais, inclusive com amostras militares. ${ }^{9,13}$ O RCO tem sido empregado como indicativo para se avaliar a gordura visceral. Assim, de acordo com o proposto por Pereira, Sichieri e de Marins, ${ }^{14}$ foi utilizado o ponto de corte de 0.95 .

Foram coletados dados de três testes funcionais: teste de resistência abdominal de um minuto, teste de flexão de braços e teste de potência aeróbia (protocolo de 12 minutos) de acordo com os protocolos propostos pela $\mathrm{FAB} .{ }^{15} \mathrm{O}$ teste de resistência abdominal consistiu em realizar o maior número possível de repetições em um minuto. O sujeito permaneceu deitado, com os braços entrelaçados estando com as mãos apoiadas nos ombros. Ao sinal de início, o sujeito realizava a flexão de abdômen, devendo encostar os cotovelos no terço distal da perna, retornando a posição inicial. O teste de flexão de braços foi realizado com os sujeitos, mantendo as mãos abertas e apoiadas ao solo, separadas na largura dos ombros, braços totalmente estendidos, mantendo o alinhamento entre tronco, quadril e pernas. Ao sinal de início, os sujeitos flexionavam os braços até formar um angulo próximo a $90^{\circ}$, retornando a posição inicial. Como resultado, foi anotada a quantidade de repetições 
corretas realizadas pelo avaliado.

O teste de 12 minutos foi realizado na pista de atletismo da organização militar. A pista de atletismo foi demarcada a cada 10 metros para facilitar a mensuração da distância alcançada no teste. Os valores foram anotados e o cálculo foi realizado pela fórmula $\mathrm{VO}_{2 \max }$ $=($ Metros $-504,9) / 44,73 .{ }^{16} \mathrm{O}$ teste de 12 minutos apresenta uma correlação moderada a elevada com o $\mathrm{VO}_{2 \max ^{\prime}}{ }^{16}$ sendo bastante utilizado em organizações militares, pela facilidade de administração, baixo custo e possibilidade de várias pessoas serem avaliadas ao mesmo tempo, 4,17,18 embora este método possua algumas ressalvas. ${ }^{19}$

\section{Análise estatística}

Para análise exploratória, recorreu-se à estatística descritiva, sendo os dados tratados em média, desvio-padrão, mínimo, máximo e percentagens para cada variável em estudo. Os dados foram analisados utilizando o software SPSS 17.0 (SPSS Inc., Chicago, EUA).

\section{RESULTADOS}

A Tabela 1 apresenta o perfil antropométrico e funcional dos sujeitos. As tabelas 2 e 3 apresentam as classificações dos estados nutricionais referentes ao IMC e ao RCQ, respectivamente.
Com base na classificação do estado nutricional, baseado na análise do IMC (tabela 2), pode-se verificar uma alta prevalência de peso normal $(75,54 \%)$, seguido de sobrepeso $(13,67 \%)$, baixo peso $(9,35 \%)$ e obesidade $(1,44 \%)$. De acordo com a Tabela 3, observa-se que 133 sujeitos apresentam RCQ menor de 0,95, índice classificado como normal e seis sujeitos são classificados com excesso de gordura abdominal.

\section{DISCUSSÃO}

O presente estudo teve como objetivo analisar o perfil morfofuncional em recrutas ingressantes em uma organização militar da FAB, no momento de seu início para cumprimento do serviço militar obrigatório. Os resultados encontrados, nesta pesquisa, evidenciam que o perfil físico dos recrutas são similares em comparação a média da população brasileira, confirmando a hipótese inicial do estudo. ${ }^{20}$ De forma geral, os sujeitos apresentaram boa condição morfológica, razoável índice de resistência abdominal, flexão de membros superiores e aptidão cardiorrespiratória. No Brasil, a prevalência populacional de sobrepeso e obesidade na população adulta está em torno de $62,5 \%$ para os homens. ${ }^{20}$

Dos recrutas estudados, $75,54 \%$ são classificados como eutróficos de acordo com o IMC. Entretanto, considera-se preocupante que $9,35 \%$ da amostra tenham baixo peso e $15,1 \%$ apresentarem excesso de

Tabela 1 - Características de antropométricas e de aptidão física em recrutas.

\begin{tabular}{|c|c|c|c|c|}
\hline & Média & Desvio Padrão & Mínimo & Máximo \\
\hline Estatura (mts) & 1,76 & 0,07 & 1,60 & 1,92 \\
\hline Massa Corporal (kg) & 67,7 & 10,5 & 48,9 & 95,2 \\
\hline Índice de Massa Corporal & 21,93 & 3,021 & 16,41 & 31,4 \\
\hline Dobra Cutânea Tricipital (mm) & 9,5 & 4,8 & 4,0 & 30,0 \\
\hline Dobra Cutânea Suprailíaca (mm) & 17,2 & 11,5 & 4,0 & 50,0 \\
\hline Dobra Cutânea Abdominal (mm) & 15,9 & 10,3 & 5,0 & 50,0 \\
\hline Gordura Corporal (\%) & 14,1 & 7,2 & 2,3 & 30,2 \\
\hline Massa Gorda (kg) & 10,1 & 6,4 & 1,2 & 27,8 \\
\hline Massa Magra (kg) & 57,6 & 6,2 & 44,6 & 72,7 \\
\hline Circunferência de Bíceps (cm) & 27 & 3 & 21 & 34 \\
\hline Circunferência de Cintura (cm) & 75 & 7 & 63 & 100 \\
\hline Circunferência de Quadril (cm) & 90 & 7 & 72 & 111 \\
\hline Relação Cintura / Quadril & 0,83 & 0,05 & 0,75 & 1,04 \\
\hline Flexão (n) & 21 & 9 & 1 & 45 \\
\hline Abdominal (n) & 35 & 8 & 11 & 56 \\
\hline Distância Percorrida - 12 minutos (mts) & 2212 & 316 & 1400 & 3000 \\
\hline VO $_{2 \max }$ Relativo (ml/kg/min) & 38,16 & 7,06 & 20,01 & 55,78 \\
\hline $\mathrm{VO}_{2 \max }$ Absoluto (I/min) & 2,56 & 0,53 & 1,43 & 4,19 \\
\hline
\end{tabular}

Tabela 2 - Classificação do estado nutricional dos recrutas em função do IMC.

\begin{tabular}{lccc}
\hline Indicador & IMC & $\mathbf{N}^{\circ}$ de Sujeitos & Percentual (\%) \\
\hline Baixo Peso & $<18,5$ & 13 & 9,35 \\
Peso Normal & $18,5-24,99$ & 105 & 75,54 \\
Sobrepeso & $25-29,99$ & 19 & 13,67 \\
Obesidade & $>30$ & 2 & 1,44 \\
\hline
\end{tabular}

Tabela 3 - Classificação do estado nutricional em função da RCQ.

\begin{tabular}{lccc}
\hline Indicador & RCQ & N $^{\mathbf{0}}$ de Sujeitos & Percentual (\%) \\
\hline Normal & $<0,95$ & 133 & 95,68 \\
Excesso de Gordura Abdominal & $>0,95$ & 6 & 4,32 \\
\hline
\end{tabular}


peso. Importante destacar que o excesso de gordura corporal está frequentemente associado a algumas alterações metabólicas e doenças crônicas, como por exemplo, diabetes, hipertensão e dislipidemias. ${ }^{21}$

As análises morfológicas foram objeto de alguns estudos e a sua relação com a capacidade física em militares de diferentes organizações. ${ }^{17,18,22,23} \mathrm{Kac}^{22}$ investigou os dados da estatura de recrutas da Marinha do Brasil nascidos entre os anos de 1940-1965. Os resultados demostraram um aumento progressivo na estatura em 0,1 cm/ano.

Analisando militares da FAB, Muniz e Bastos ${ }^{9}$ avaliaram a prevalência da obesidade e suas implicações na medicina aeroespacial. Foram avaliados 28.745 militares de 174 Organizações Militares da FAB, correspondendo a $41,68 \%$ de todo o efetivo. De acordo com os resultados baseados no IMC, $53,3 \%$ dos militares encontram-se com peso normal, $1,2 \%$ com baixo peso e $45,5 \%$ com sobrepeso ou obesidade. Comparando estes dados com o presente estudo (tabela 2), observam-se no presente estudo valores inferiores de militares com sobrepeso ou obesos.

Resultado semelhante ao presente estudo foi analisado por Neves ${ }^{3}$ que investigou a associação entre o IMC, a RCQ e a circunferência de cintura com a prevalência de hipertensão arterial sistêmica em militares do sexo masculino. Os resultados deste estudo apontam que houve $12,91 \%$ dos sujeitos com classificação de obesos ou com excesso de gordura. Considerando o IMC, foi revelado que $7,98 \%$ estavam com a mesma classificação considerando a RCQ. A RCQ foi diagnosticada também como o indicador antropométrico com melhor associação com a pressão arterial sistêmica. ${ }^{3}$ Estes resultados são similares ao presente estudo, considerando o IMC $(15,11 \%$ classificados com sobrepeso ou obesos) e a RCQ $(4,32 \%$ com excesso de gordura abdominal).

A obesidade é epidemia no mundo moderno e aumenta a um ritmo alarmante, com grandes consequências humanas e econômicas. ${ }^{24}$ Para os milhões de indivíduos com excesso de peso e moderadamente obesos, a principal forma de tratamento da obesidade é a melhora no estilo de vida, com modificações na dieta e na prática de atividade física. ${ }^{25}$

Com o propósito de investigar o nível de aptidão física e de adiposidade corporal em policiais militares, Santos et al. ${ }^{17}$ concluíram que $60,8 \%$ dos militares foram classificados com excesso de peso (de acordo com o (MC), valores muito superiores ao do presente estudo. Cabe-se destacar que nesta amostra, a idade média dos sujeitos foi muito superior ao do presente estudo (30 $\pm 3,97$ anos). Na avaliação cardiorrespiratória, observou-se que o $\mathrm{VO}_{2 \max }$ teve valor médio de 42,1 44,5 $\mathrm{ml} \cdot \mathrm{kg}^{-1} \cdot \mathrm{min}^{-1}$, valores superiores ao do presente estudo $\left(38,10 \pm 7,06 \mathrm{ml} \cdot \mathrm{kg}^{-1} \cdot \mathrm{min}^{-1}\right)$.

Hage e Reis Filho $^{18}$ analisaram a mudança no desempenho físico e o perfil antropométrico em aluno da Policia Militar do Estado de Mato Grosso após 12 semanas de treinamento físico. Os achados deste estudo foram similares ao presente estudo apenas na variável estatura. Na massa corporal e no IMC observaram-se valores superiores. Entretanto, analisando os testes de flexão de braço, corrida de 12 minutos e os valores de $\mathrm{VO}_{2 \text { max }}$ observam-se melhores resultados $(34,4 \pm 4,3$ vs $21 \pm 9$ (n); $2572,1 \pm 261,6$ vs $2212 \pm 316$ (mts) e $45,9 \pm 5,8$ vs $38,16 \pm 7,06\left(\mathrm{ml}^{\mathrm{kg}}{ }^{-1} \cdot \mathrm{min}^{-1}\right)$, respectivamente) quando comparado ao presente estudo. No entanto, devem-se considerar as diferenças de idade dos sujeitos entre os dois estudos.

O ponto de corte proposto pelo FITNESSGRAM, para estimar a saúde cardiovascular fixa o limiar de 42 $\mathrm{ml} . \mathrm{kg}^{-1} \cdot \mathrm{min}^{-1}$, como sendo adequado para o indicativo de risco futuro. Assim, com base nos dados, a prevalência de recrutas com um risco cardiovascular (definido pela capacidade aeróbia) é cerca de $68 \%$. Isto representa mais da metade de todos os avaliados, requerendo atenção especial dos gestores para alterar este ruim indicativo acima exposto.

De acordo com achados anteriores, a inatividade física atinge aproximadamente $26,3 \%$ da população brasileira.26 Torna-se relevante a intervenção física adequada em consonância com a melhora da ingestão alimentar (reeducação), para o desenvolvimento da aptidão física e consequentemente redução dos índices de obesidade e melhoria da qualidade de vida, em especial dos recrutas avaliados no presente estudo.

\section{CONSIDERACÕES FINAIS}

Considera-se preocupante a uma parte da amostra ( 25\%) com peso inadequado (baixo peso ou sobrepeso) e a baixa capacidade aeróbia nos recrutas ingressantes na unidade militar. Sugere-se assim que sejam realizadas intervenções adequadas com planejamento e prescrição de exercícios físicos, bem como uma adequada ingestão alimentar com vistas a reduzir os malefícios relacionados ao peso inadequado e a baixa capacidade cardiorrespiratória, tendo em vista que as atividades militares necessitam de excelentes níveis de aptidão física, para o cumprimento das missões ao qual lhes são impostas.

\section{REFERÊNCIAS}

1. Fulton JE, Garg M, Galuska DA, Rattay KT, Caspersen CJ. Public health and clinical recommendations for physical activity and physical fitness. Sports Medicine 2004;34(9):581-599

2. Rocha CRGS, Freitas CR, Comerlato M. Relação entre nível de atividade física e desempenho no teste de avaliação física de militares. Revista de Educação Física 2008;142(3):19-27.

3. Neves EB. Prevalência de sobrepeso e obesidade em militares do exército brasileiro: associação com a hipertensão arterial. Ciên Saúde Coletiva 2008;13(5):1661-8.

4. Mendes LCV, Ferreira CES. Comparação de dois protocolos indiretos na avaliação da capacidade aeróbia de alunos do núcleo de preparação de oficiais da reserva. Educação Física em Revista 2010:4(2).

5. Brasil. Ministério da Saúde. Cadernos de Atenção Básica, n. 12. Série A. Normas e Manuais Técnicos. Acessado 18 de Janeiro de 2016. Disponível em: http://dtr 2004.saude.gov. br/nutricao/documentos/doc_obesidade.pdf

6. Rodrigues AN, Perez AJ, Carletti L, Bissoli NS, Abreu GR. Valores de consumo máximo de oxigênio determinados pelo teste cardiopulmonar em adolescentes: uma proposta de classificação. Jornal de Pediatria 2006;82(6):426-430. 
7. ACSM - American College of Sports Medicine. Manual do ACSM para avaliação da aptidão física relacionada à saúde. Rio de Janeiro: Guanabara Koogan; 2006.

8. Ortega FB, Ruiz JR, Castillo MJ, Sjöström M. Physical fitness in childhood and adolescence: a powerful marker of health. International Journal of Obesity 2008;32(1):1-11.

9. Muniz GR, Bastos FIPM. Prevalência de obesidade em militares da força aérea brasileira e suas implicações na medicina aeroespacial. R. Educ Tecn Apl Aeron 2010;2(1):25-36.

10. Perini TA, Oliveira GLD, Ornellas JDS, Oliveira FPD. Cálculo do erro técnico de medição em antropometria. Revista Brasileira de Medicina do Esporte 2005;11(1):81-5.

11. Guedes DP. Composição Corporal: Princípios Técnicas e Aplicações. Ed. APEF - Londrina, $2^{\mathrm{a}}$ ed., 1994.

12. Siri WE. Body composition from fluid spaces and density. In: Brozek J, Henschel A, editors. Techniques for measuring body composition. Washington DC: National Academy of Science 1961:223-44.

13. Hilgenberg FE, Santos AS, Carvalho A, Silveira EA, Cominetti C. Cardiovascular risk factors and food consumption of cadets from the Brazilian Air Force Academy. Ciência \& Saúde Coletiva 2016;21(4):1165-1174.

14. Pereira RA, Sichieri R, de Marins VMR. Razão cintura/quadril como preditor de hipertensão arterial. Cad Saúde Pública 1999;15:333-344.

15. Brasil. Ministério da Defesa. ICA 54-1: teste de avaliação do condicionamento físico no comando da Aeronáutica. Brasília, DF: Comando da Aeronáutica/ Educação Física e Desportos, 2011.

16. Cooper $\mathrm{KH}$. A means of assessing maximal oxygen intake. Correlation between field and treadmill testing. JAMA 1968;203(3):201-204

17. Santos JAB, Silva RG, Browne RAV, Sales MM, Russo AF, Segundo PR. Nível de adiposidade corporal e de aptidão física de Policiais Militares de Boa Vista-Roraima. Revista Brasileira de Prescrição e Fisiologia do Exercício 2013;7(37).

18. Hage CC, Reis Filho AD. Análise do desempenho físico e perfil antropométrico dos alunos do 28 curso de formação de soldados da PM/MT-CEsp após 12 semanas de treinamento físico. Revista Brasileira de Prescrição e Fisiologia do Exercício 2013:7(41).

19. Kravchychyn ACP, Alves JCC, Kravchychyn TP, Nogueira GÂ, Machado FA. Comparison between the direct and indirect methods of VO2máx determination in runners. Revista Brasileira de Medicina do Esporte 2015;21(1):17-21.

20. IBGE - Instituto Brasileiro de Geografia e Estatística. Pesquisa de orçamentos familiares 2008-2009: despesas, rendimentos e condições de vida. Rio de Janeiro: IBGE; 2010. Disponível em: http://www.ibge.gov.br/home/estatistica/populacao/ condicaodevida/pof/2008_2009/default.shtm.

21. Rezende FAC, Rosado, LEFPL, Priore SE, Franceschini SDCC. Aplicabilidade de equações na avaliação da composição corporal da população brasileira. Revista de Nutrição 2006;19(3):357-367.

22. Kac G. Secular trends in the stature of Brazilian Navy recruits born from 1940 to 1965. Cadernos de Saúde Pública 1998:14(3);565-573.

23. Okamura $A B$, Pinheiro LRL, Berton G, Okamura AMNC, Garcia AA, Mello DB. Aptidão neuromuscular de militares da Força de Pacificação do Exército Brasileiro nos Complexos do Alemão e da Penha. Revista de Educação Física 2016;85(1).

24. Cawley J, Meyerhoefer C. The medical care costs of obesity: An instrumental variables approach. Journal of health economics 2012;31(1):219-230.

25. Low AK, Bouldin MJ, Sumrall CD, Loustalot FV, Land KK. A clinician's approach to medical management of obesity. The American Journal of the Medical Sciences 2006;331(4):175-182.

26. Brasil, Ministério da Saúde. VIGITEL: vigilância de fatores de risco e proteção para doenças crônicas por inquérito telefônico. Brasília: Ministério da Saúde; 2009.

Como citar: CAMPOS, Leandra Cristina Benetti et al. Avaliação do perfil morfofuncional de recrutas ingressantes em uma unidade militar da força aérea brasileira. Cinergis, Santa Cruz do Sul, v. 17, n. 2, jun. 2016. ISSN 2177-4005. Disponível em: <https://online.unisc.br/seer/index.php/cinergis/article/view/7585>. Acesso em: 04 jul. 2016. doi:http://dx.doi. org/10.17058/cinergis. v17i2. 7585. 